\title{
Aplicando a Metáfora dos Cadernos Escolares Digitais para Apoiar o Processo Colaborativo de Resolução de Exercícios de Lógica
}

\author{
Fabricio Costa da Silva', Maiglon Alexandre Lubacheuski' ${ }^{1}$, Matheus Campezatto \\ Galão ${ }^{1}$, João Carlos Gluz ${ }^{1}$ \\ ${ }^{1}$ Universidade do Vale do Rio dos Sinos - São Leopoldo - RS - Brazil \\ ${ }^{2}$ IBCMED - Porto Alegre - RS - Brazil \\ fabriciocsilva@yahoo.com.br,maiglonl@gmail.com, matheusz.cg@gmail.com, \\ jcgluz@gmail.com
}

\begin{abstract}
This work assumes the premise that notebooks used in class can be implemented digitally, not only to register facts, ideas, doubts and difficulties encountered in day to day learning activities, but also to help organize contents and serve as a means of interaction and collaboration between groups of students and between students and teachers. From this, the paper proposes a web-based tool that offers a free space where students can take notes, solve exercises, store multimedia educational materials and documents, exchange information and interact with colleagues and teachers. The tool is aimed at the domain of Logic, being integrated to a learning environment that offers intelligent tutoring to solve problems in this discipline. The article makes a comparative analysis of the state of the practice regarding the web software for electronic notebooks that currently exists. Main characteristics of the computational model of the proposed tool are also presented. Results of experimental validation of the tool are shown at the end of the paper.
\end{abstract}

Resumo. Este trabalho assume a premissa que simples caderno escolares podem ser implementada digitalmente de forma a não apenas registrar fatos, idéias, dúvidas e dificuldades encontradas no dia a dia da aprendizagem, mas também ajudar na organização dos conteúdos e servir como um meio de interação e colaboração entre grupos de alunos e entre alunos e professores. Para tanto, o trabalho propõe uma ferramenta baseada na web que oferece um espaço livre onde alunos podem fazer anotações, resolver exercícios, armazenar materiais educativos multimídia e documentos, intercambiar informações e interagir com colegas e professores. A ferramenta está voltada para o domínio de ensino de Lógica, sendo integrada a um ambiente de ensino que oferece tutoria inteligente para a resolução de problemas nesta disciplina. $O$ artigo faz uma análise comparativa do estado da prática relativa aos softwares web para cadernos eletrônicos existentes atualmente. Após são apresentadas as principais características do modelo computacional da ferramenta proposta. Ao final são apresentados os resultados da validação experimental dessa ferramenta.

\section{Introdução}

Com o avanço da internet e das facilidades advindas do uso das tecnologias digitais, o 
VII Congresso Brasileiro de Informática na Educação (CBIE 2018)

Anais do XXIX Simpósio Brasileiro de Informática na Educação (SBIE 2018)

ensino tradicional vem cada vez mais se adaptando a uma forma híbrida de ensino (blended learning, ou b-learning) que incorpora tanto aulas presenciais como um suporte digital ubíquo [Garrison, 2004; Güzer, 2014]. Novos métodos de ensino, como a sala de aula invertida (flipped classroom) [Bergmann e Sams, 2012], também se beneficiam do b-learning. Plataformas digitais educacionais e ambientes virtuais de aprendizagem são as ferramentas usuais para intermediar processos de b-learning, permitindo disponibilizar ao aluno, conteúdos e atividades vistas em sala de aula, idealmente ajudando-o em sua busca por conhecimento e tornando-o coautor no processo de ensino-aprendizagem. Nesse contexto, cadernos eletrônicos têm sido utilizados como uma forma de organizar e distribuir digitalmente conteúdos e conhecimentos de vários domínios educacionais. Esse uso como formato de distribuição de conteúdos é bastante comum atualmente, podendo ser reconhecido como a base de programas de cadernos eletrônicos adotada por Secretarias Estaduais de Educação no Brasil [SEE/SP, 2014; Filipouski, 2009]. Porém o uso dos cadernos apenas para distribuição de conteúdos digitais ignora as possibilidades que a metáfora do caderno escolar pode oferecer para fins educacionais no contexto do b-learning (e também no contexto da educação à distância). Aqui entra a importância do uso de metáforas no design de interfaces com o usuário, o que, para Hiratsuka (1996, p. 9), "permite maiores índices de produtividade, pois facilitam a sua operacionalidade, além de facilitarem o seu aprendizado por usuários novatos". Essas possibilidades não têm sido exploradas nos atuais cadernos eletrônicos, que são basicamente livros ou apostilas eletrônicas.

Mas o que a metáfora de Caderno Escolar Digital (CED) poderia oferecer? Neste trabalho partimos da idéia que CED nada mais é que a implementação e suporte digital das várias funcionalidades que um caderno escolar (um caderno de aula comum nãodigital) oferece à alunos e professores. Cadernos de aula são instrumentos muito úteis para a aprendizagem, sendo extensivamente usados desde que materiais leves, baratos e duráveis para registro da escrita foram inventados (papel inventado pelos Árabes, desde a Idade Média, ou o papiro, durante a Antiguidade Clássica). Dentre outras possibilidades, cadernos servem para anotar material visto em aula, são usados para armazenar materiais fornecidos pelo professor, registram a resolução de exercícios e atividades, tanto de exemplos vistos em aula, quanto resolvidos pelo aluno. Esses cadernos registram fatos, idéias, dúvidas e dificuldades encontradas no dia a dia do processo de aprendizado. Além de obviamente serem usados de forma individual, também servem como veículos de colaboração e compartilhamento de informações e conhecimentos tanto entre alunos quanto entre professores e alunos.

Assim, um CED tem que ser capaz de implementar essas (e talvez algumas outras) funcionalidades vistas acima, possibilitando o registro dos processo de aprendizagem pelo aluno, ajudando na organização do conteúdo e também servindo como um meio de interação entre alunos e entre estes e os professores, através do compartilhamento de lições e materiais. $O$ fato de serem digitais permite a estes cadernos assumir novas feições. Por exemplo, um CED voltado à resolução de problemas de Matemática pode possibilitar que o aluno resolva exercícios, à medida em que faça anotações e tire dúvidas com o professor, possibilitando ainda que o professor transfira materiais extras no intuito de auxiliar o aluno a busca por si soluções para os problemas.

De um ponto de vista mais geral, pode-se argumentar que os CED se relacionam ao conceito de transmídia [Castells, 1999], trazendo a possibilidade que o conteúdo 
VII Congresso Brasileiro de Informática na Educação (CBIE 2018)

Anais do XXIX Simpósio Brasileiro de Informática na Educação (SBIE 2018)

educacional inclua vários tipos de informações, em vários formatos, não apenas na presença física do professor explanando o conteúdo presencialmente, mas incluindo o uso de desenhos, gráficos, fotografias, vídeos, etc. Santaella (2007) aborda a questão da transcendência das relações tradicionais entre professor autor e o aluno usuário, bem como entre o espaço e o tempo. O uso dos CED pode abrir um espaço nessa relação tradicional ao trazer a possibilidade de cocriação, ou seja, alunos e professores podem, ao mesmo tempo, criar conhecimento. Nesse sentido, os CED se tornam, mais que um mecanismo de distribuição de conteúdos, um veículo para o intercâmbio de informações e ideias, onde todos podem participar de maneira ativa na busca pelo conhecimento.

Seguindo essa visão, o presente trabalho apresenta uma ferramenta de suporte a CED, denominado de Logos Workbook, que é baseada na web e oferece um espaço livre onde o aluno pode fazer anotações, resolver exercícios, armazenar materiais educativos multimídia e documentos, intercambiar informações e interagir com colegas e professores. Esta ferramenta foi inicialmente avaliada no domínio de ensino de Lógica, tendo sido completamente integrada ao ambiente inteligente de ensino de Lógica Heráclito [Gluz et al., 2017]. O domínio de Lógica foi escolhido não apenas porque a Lógica é uma disciplina fundamental para o currículo das áreas científicas e tecnológicas (genericamente agrupadas sob a sigla STEM - Science, Technology, Engineering, and Mathematics), mas também por ser um domínio de notória dificuldade de aprendizagem e assim se configurar como um bom campo de prova ao Logos Workbook.

Assim o objetivo imediato do Logos Workbook foi trazer uma interface de documentos dinâmicos, ativos e inteligentes para os alunos que utilizem o ambiente Heráclito. Este ambiente oferece tutoria inteligente para a resolução de problemas de Lógica, possibilitando que o aluno possa, por si mesmo, descobrir onde está errando na resolução destes problemas e como pode avançar nesse processo de resolução. Além disso o Heráclito também disponibiliza uma ferramenta de apoio ao professor, denominada Saapiens [Rodrigues et al., 2014] que, através de uma interface de dashboard, oferece uma visão global dos cadernos do Logos Workbook. Com a integração do Logos Workbook ao Saapiens os professores podem criar e publicar conteúdos e atividades educacionais nos cadernos digitais de alunos individuais, grupos de alunos ou de toda a turma. Através de mecanismos de learning analytics implementados no Saapiens, capazes de analisar os registros contidos nos cadernos no Logos Workbook, os professores podem acompanhar o processo de aprendizagem de forma a detectar as maiores dificuldades encontradas por alunos grupos de alunos ou de toda a turma e com isso identificar pontos que precisam de maior atenção.

$\mathrm{O}$ artigo apresenta uma análise do estado da prática relativa aos softwares web existentes atualmente que podem oferecer suporte para CED, comparando-os com as características da ferramenta proposta neste trabalho. A seguir são apresentadas as principais características do modelo computacional do Logos Workbook. No final do trabalho são apresentados os resultados da validação experimental da ferramenta.

\section{Trabalhos Relacionados}

O uso de CED, particularmente aqueles baseado na web, é uma tendência recente em $b$ learning, porém a literatura científica sobre esse tema ainda é limitada. O trabalho [Albrecht, 2017] é um dos poucos exemplos dessa tendência, oferecendo uma discussão e análise do uso de CED baseado no software Apache Zeppelin, na Educação Superior 
VII Congresso Brasileiro de Informática na Educação (CBIE 2018)

Anais do XXIX Simpósio Brasileiro de Informática na Educação (SBIE 2018)

em Ciência da Computação. Sendo assim, foi feita uma análise do estado da prática que pesquisou ambientes e sistemas web existentes atualmente que podem ser usados para suportar os CED. Posteriormente, foi feita uma analise comparativa desses softwares com a ferramenta proposta neste artigo. Os softwares analisados são descritos a seguir.

O Jupyter Notebook (http://jupyter.org/) e o Apache Zeppelin (https://zeppelin.apache.org/) são aplicativos web para criação e compartilhamento de documentos ativos, que contém conteúdos estáticos textuais e gráfico e conteúdos dinâmicos que suportam código de programação ativo, manipulação e visualização de equações, visualização e transformação de dados, simulação numérica e modelagem estatística. Ambos sistemas são usados para criação de modelos analíticos de dados, mas o Jupyter Notebook também tem um bom suporte para desenvolvimento de programas. Por outro lado esses sistemas não suportam mecanismos de interação síncrona ou assíncrona por meio dos seus cadernos de anotações (notebooks).

O Evernote (https://evernote.com/) é um software gratuito que permite a criação de cadernos de notas com conteúdos estáticos como imagens, arquivos, checklists, lembretes e anotações. O Evernote permite a interação entre seus usuários através de um serviço de chat, sem a necessidade de um segundo software para isso. É possível também compartilhar o caderno com um grupo. O OneNote (https://www.onenote.com) suporta cadernos digitais para criação de conteúdos estáticos como textos, tabelas, imagens, arquivos, áudios e desenhos. Oferece a possibilidade de colaboração multiusuário pelo compartilhamento e edição conjunto de caderno com outros usuários, com as alterações aparecem em tempo real.

O Google Classroom (https://classroom.google.com) é um serviço que tem por objetivo ajudar professores e alunos a interagirem entre si e melhorarem a qualidade de suas aulas. A comunicação no Google Classroom é assíncrona e de via dupla entre alunos e professores. Professores podem criar conteúdos estáticos para a turma, como textos e vídeos, além de materiais dinâmicos como questionários. Os alunos acompanham materiais e tarefas da turma criada pelo professor, interagem com demais alunos pelo mural (ou email, externo à ferramenta), compartilham recursos, enviam tarefas propostas e recebem avaliações e feedback pelo do professor. O Maple (https://www.maplesoft.com/products/Maple/) é um ambiente computacional simbólico e matemático, cobrindo aspectos da computação técnica, tais como análise de dados, computação matricial, conectividade e visualização. Segundo Heal et al. (1998) e Monagan et al. (1998) o objetivo do Maple é ser um provador de teoremas equacionais, mas que pode ser usado nas área de Computação Científica, Matemática, Física, Química e similares áreas técnicas. O Maple também possibilita o desenvolvimento de materiais didáticos interativos, cujo objetivo principal é garantir autonomia aos alunos para que criem situações específicas de aprendizagem.

A Tabela 1 apresenta um comparativo entre o Logos Workbook e os softwares descritos acima. Os critérios usados na comparação foram: CE - suporte a conteúdos estáticos, como vídeos, textos, imagens; CD - suporte a conteúdos dinâmicos, como simulações, cálculos numéricos e simbólicos, exercícios iterativos; CI - suporte a conteúdos inteligentes, que são conteúdos dinâmicos como exercícios iterativos, acompanhados por um serviço automático e inteligente de tutoria, uma implementação parcial desse conceito é possível pela programação explícita de um modelo computacional inteligente e sua posterior incorporação ao caderno como um conteúdo 
VII Congresso Brasileiro de Informática na Educação (CBIE 2018)

Anais do XXIX Simpósio Brasileiro de Informática na Educação (SBIE 2018)

dinâmico; DC - voltado a domínios específicos de conhecimento ou genérico; AA integração com ambiente digital ou virtual de aprendizagem; VE - software voltado especificamente para Educação; IC - suporte para interação/comunicação entre os usuários, pode ser tanto síncrona, com chats, ou assíncrona, com murais ou quadros de avisos; CC - suporte a compartilhamento e edição colaborativa de cadernos.

Tabela 1. Análise comparativa dos software para CED baseados na web

\begin{tabular}{|l|c|l|l|l|l|l|l|l|}
\hline Software & CE & CD & \multicolumn{1}{|c|}{ CI } & \multicolumn{1}{|c|}{ DC } & AA & VE & IC & CC \\
\hline Jupyter & $\operatorname{sim}$ & $\operatorname{sim}$ & parcial & $\begin{array}{l}\text { Comput., Matemat., } \\
\text { Estatística }\end{array}$ & não & não & não & não \\
\hline Zeppelin & $\operatorname{sim}$ & $\operatorname{sim}$ & parcial & $\begin{array}{l}\text { Comput., Matemat., } \\
\text { Estatística }\end{array}$ & não & não & não & não \\
\hline Evernote & $\operatorname{sim}$ & não & não & genérico & não & não & sínc. & sim \\
\hline Onenote & $\operatorname{sim}$ & não & não & genérico & não & não & assíncr. & sim \\
\hline Google Classroom & $\operatorname{sim}$ & $\operatorname{sim}$ & não & genérico & sim & sim & assínc. & não \\
\hline Maple & $\operatorname{sim}$ & $\operatorname{sim}$ & parcial & $\begin{array}{l}\text { Comput., Matemat., } \\
\text { Estatística }\end{array}$ & não & não & não \\
\hline LOGOS Workbook & $\operatorname{sim}$ & $\operatorname{sim}$ & $\operatorname{sim}$ & Lógica & sim & sim & assínc. & sim \\
\hline
\end{tabular}

Pela comparação apresentada na Tabela 1, pode-se observar que o Logos Workbook se destaca dos demais softwares por ser um CED exclusivo para o ensino de Lógica, mas que suporta além de conteúdos estáticos e dinâmicos, conteúdos inteligentes capazes de ajudar na resolução orientada de problemas. Além disso este sistema possibilita a tanto a interação assíncrona quanto o trabalho colaborativo entre professores e alunos e entre os colegas de um grupo de alunos, diretamente por meio cadernos.

\section{Interface e Modelo Arquitetural}

A interface do Logos Workbook emprega a metáfora de caderno, tanto na aparência, exibindo linhas na tela e paginação em "folhas", bem como no ideal de que o usuário tem controle sobre o conteúdo, podendo inserir anotações cujo texto é livre, resolver um exercício de Lógica ou submeter uma dúvida ao professor, como melhor aprouver.

Os cadernos do Logos Workbook estão divididos por disciplinas e turmas, sendo de propriedade dos seus usuários. Os usuários estão divididos em dois papeis principais: alunos e professores. Independente do tipo de usuário, as interações com o caderno são registradas por blocos de conteúdos ordenados cronologicamente, que são de propriedade do usuário, podendo ser excluídos ou alterados somente quando este o desejar. Os blocos podem ser classificados como públicos ou privados. Somente blocos públicos são visíveis aos outros usuários que têm acesso ao caderno. O professor tem acesso aos cadernos de sua turma, podendo postar nesses cadernos blocos para apresentar conteúdos, disponibilizar materiais, listas de atividades e exercícios, inserir avisos e comentários e interagir com as dúvidas dos alunos. Grupos de colegas também podem postar blocos de anotações, comentários e exercícios no caderno de um aluno, caso tenham o acesso permitido por esse aluno ao seu caderno. Porém, tanto professores quanto colegas não podem alterar os blocos de propriedade do aluno. Blocos dos colegas podem ser excluídos do caderno do aluno, porém blocos dos 
VII Congresso Brasileiro de Informática na Educação (CBIE 2018)

Anais do XXIX Simpósio Brasileiro de Informática na Educação (SBIE 2018)

professores, não podem ser apagados pelo aluno, ainda que este possa filtrar a exibição de blocos para ver, por exemplo, apenas seus blocos, ou os blocos de colegas ou do professor. A Figura 1 apresenta a interface tal como exibida em um navegador executando em um computador desktop. A Figura 1(a) o mostra um exemplo de caderno escolar e a Figura 1(b) a interface de edição de um bloco de anotações.

As possibilidade de uso dos cadernos no Logos Workbook será ilustrada através de um cenário de uso. Inicialmente o aluno irá registrar anotações acerca do conteúdo estudado de tabelas-verdade, fórmulas contingente, tautologias e contradições. Para tanto, basta abrir seu caderno no Logos Workbook e na tela inicial clicar na opção "Anotação", que uma janela de edição similar à apresentada na Figura 1(b), será mostrada na tela. Nesta janela de anotação pode ser inserido um título e haverá à disposição uma caixa de texto onde o aluno poderá fazer suas anotações da maneira como melhor lhe aprouver, indicando destaques no texto, dividindo-o em títulos e subtítulos, etc. Ao encerrar a anotação, ela aparecerá na tela do caderno como o primeiro item, uma vez que o caderno está organizado cronologicamente, facilitando a localização do conteúdo.

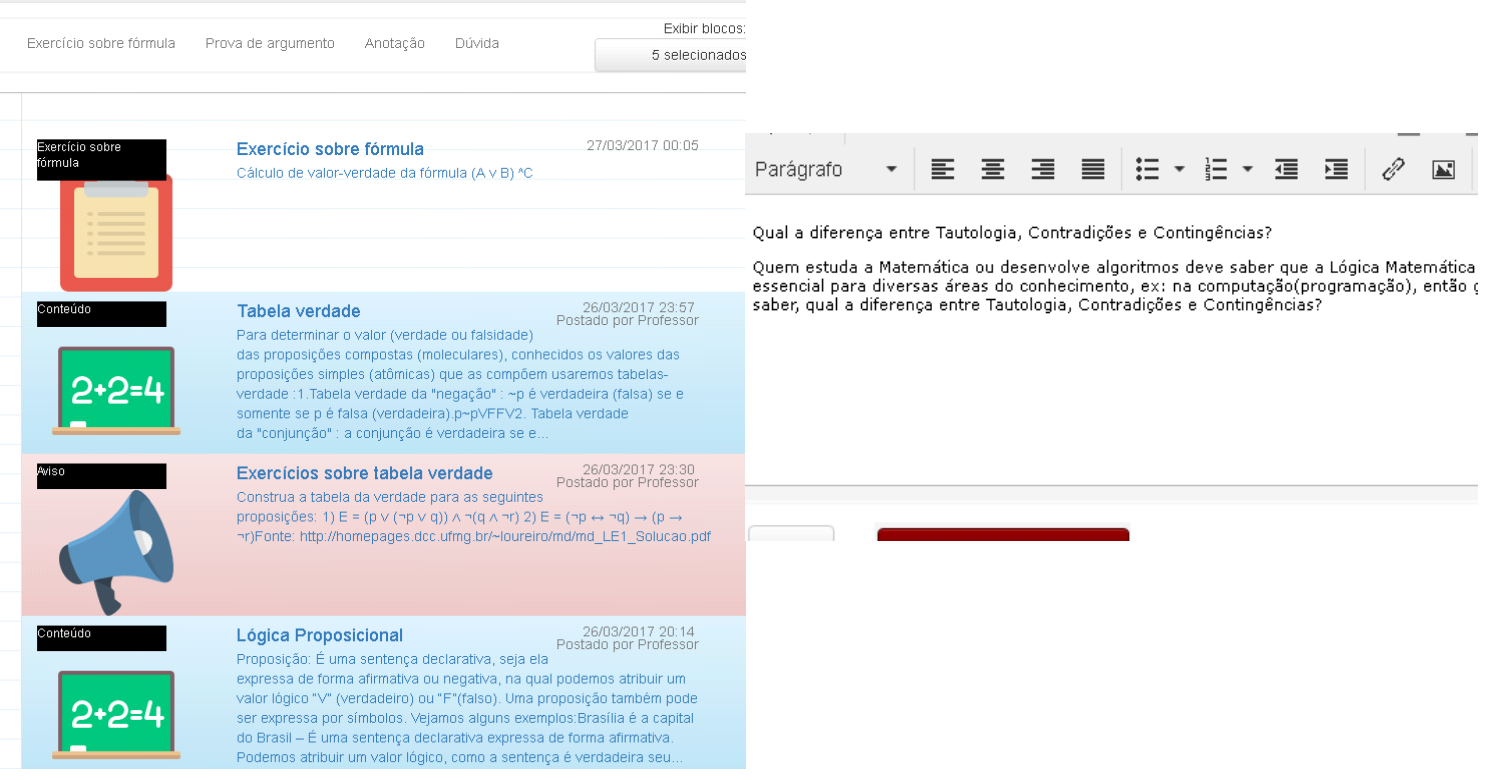

(a) Tela inicial do caderno

(b) Bloco de anotações

Figura 1. Tela inicial e bloco de anotações do caderno Logos Workbook

$\mathrm{Na}$ continuação, o aluno precisa interagir com o professor para elucidar dúvidas sobre tautologias e contradições. Para se comunicar com o professor, o aluno deverá clicar no botão "dúvida", no menu do caderno Logos Workbook que uma nova tela será aberta contendo uma caixa de texto similar ao bloco de anotações mostrado na Figura 1(b), para que o aluno possa escrever sua dúvida a ser respondida pelo professor. Quando o aluno terminar de escrever sua dúvida, deverá clicar no botão "enviar" para que a dúvida fique disponível para o professor. A Figura 2(a) mostra como esta dúvida fica registrada no bloco de dúvidas, quando aberto pelo professor. Neste bloco o professor tem um espaço disponível para responder aos questionamentos do aluno, facilitando a comunicação entre eles e possibilitando a elucidação de dúvidas de maneira rápida. Na Figura 2(b) é apresentada uma tela com a resposta enviada para a dúvida do aluno. 
VII Congresso Brasileiro de Informática na Educação (CBIE 2018)

Anais do XXIX Simpósio Brasileiro de Informática na Educação (SBIE 2018)

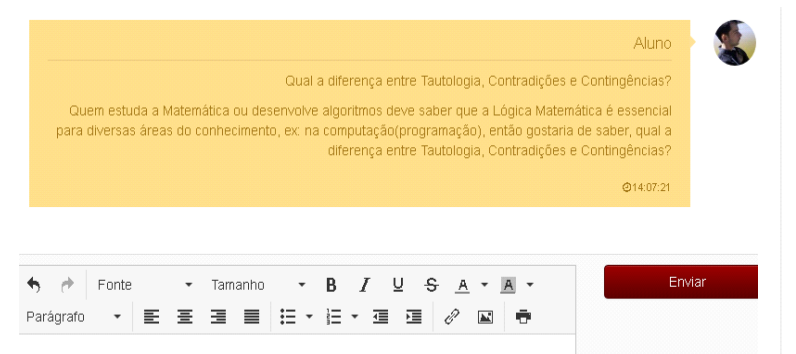

(a) Dúvida enviada pelo aluno

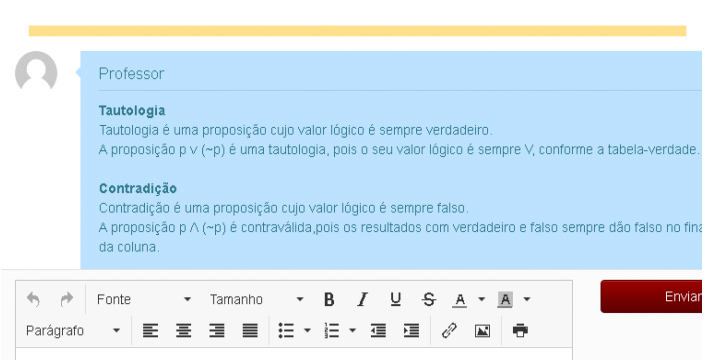

(b) Resposta do professor

Figura 2. Telas demonstrando a interação pode meio de blocos de dúvida

Para permitir que o aluno possa exercitar um pouco mais seus conhecimentos de sobre fórmulas contingentes, tautologias e contradições o professor posta no caderno do aluno uma lista exercício de resolução de tabela-verdade. A Figura 3 mostra a janela da ferramenta de resolução de exercícios de tabela-verdade que está integrada ao Logos Workbook e que é ativada quando o aluno clica em um exercício de tabela-verdade.

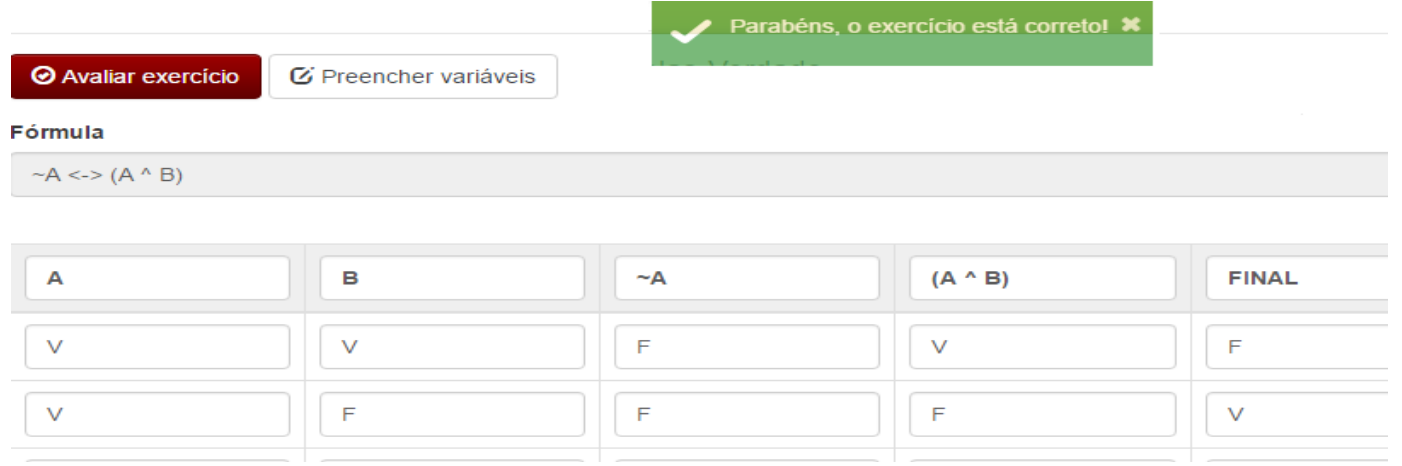

Figura 3. Ferramenta de resolução de tabelas-verdade integrada ao Logos Workbook

O modelo computacional do Logos Workbook foi projetado como uma arquitetura de software assumindo uma divisão em camadas de apresentação, integração, aplicação e persistência tal como apresentada na Figura 4.

Cada uma dessas camadas possui uma responsabilidade distinta: a) Camada de Apresentação: implementa a interface visual compatível com a web, e é responsável por interagir com o usuário, exibindo as informações manipuladas pelas camadas inferiores e responsabilizando-se pelos eventos de entrada, como cliques do mouse e acionamentos do teclado. O modelo adotado emprega Bootstrap para oferecer responsividade e AngularJS como framework de desenvolvimento JavaScript. b) Camada de Aplicação: é responsável pelo serviço inteligente de tutoria provido ao aluno e pelo serviço de acompanhamento pedagógico, autoria de conteúdos e planejamento curricular fornecido ao professor (não é descrito neste artigo). c) Camada de Persistência: contém as interações necessárias para integrar o banco de dados com os objetos de negócio, isolando a linguagem de consulta e fornecendo acesso simplificado dos dados para as camadas superiores. Os cadernos e as interações dos alunos são representadas como documentos JSON e armazenadas usando MongoDB. d) Camada de Integração: interconecta as camadas de aplicação e persistência a camada de apresentação através de web services. 
VII Congresso Brasileiro de Informática na Educação (CBIE 2018)

Anais do XXIX Simpósio Brasileiro de Informática na Educação (SBIE 2018)

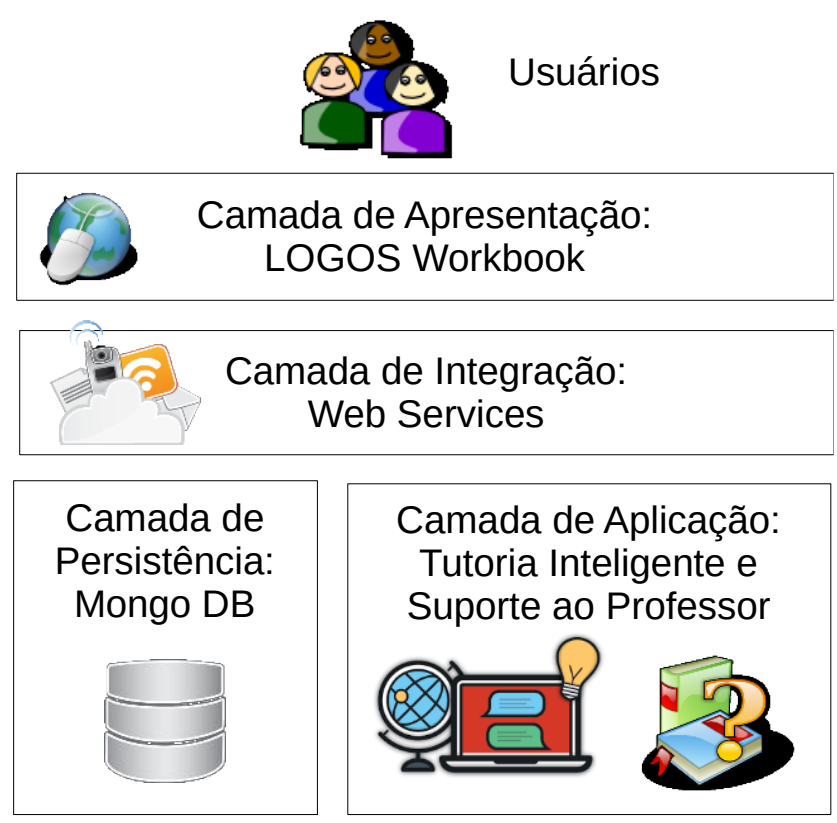

Figura 4. Arquitetura em camadas do LOGOS Workbook

\section{Experimentos e Resultados}

Para avaliar a contribuição educacional do Logos Workbook, bem como apurar sua usabilidade, realizou-se um experimento de avaliação da usabilidade deste sistema com alunos de Computação de nossa universidade. O experimento foi realizado em dois dias e contou com uma amostra total de 5 alunos participantes, escolhidos por conveniência. Todos os alunos estão cursando Ciência da Computação, são do sexo masculino, e têm entre 20 e 30 anos. Nenhum aluno tinha experiência prévia com o uso de ferramentas ou ambientes de ensino que suportassem cadernos digitais.

Para sua realização, cada aluno recebeu um roteiro de atividades para realizar no Logos Workbook. Este roteiro engloba 11 atividades que exploram os principais recursos do caderno escolar digital. Dentre essas atividades foi solicitada a resolução de quatro exercícios de Lógica com o suporte do Logos Workbook: a decomposição da fórmula $A->(B \vee A)$ em suas variáveis elementares, a avaliação do valor-verdade dessa fórmula para $A=V$ e $B=F$, a construção da tabela verdade da fórmula $\sim(A \wedge \sim B)$ e a prova formal da validade do argumento lógico $\sim \mathrm{A}, \mathrm{B}->\mathrm{A} \mid-\sim \mathrm{B}$.

Após a realização do experimento foi conduzida uma avaliação da usabilidade e de utilidade do caderno eletrônico, de acordo com a percepção dos alunos participantes. Esta avaliação foi efetuada através de um questionário elaborado com base na metodologia TAM2 (Technology Acceptance Model, Version 2) [Marangunić, 2015, p. 81-95] que avalia duas duas variáveis: a utilidade e a facilidade de uso percebida, que serão utilizadas como critério para as questões. O questionário foi composto de 18 questões projetadas para avaliar o nível de concordância dos usuários com ideias expressas através de afirmações diretas, conforme [Nemoto, 2014, p. 1-8], e tiveram suas respostas medidas através da Escala Likert, conforme [Bertram, 2016], sendo a seguir analisadas. Das 18 questões, 15 foram elaboradas para avaliar a facilidade de uso da ferramenta e 3 para avaliar a percepção de utilidade desta. 
VII Congresso Brasileiro de Informática na Educação (CBIE 2018)

Anais do XXIX Simpósio Brasileiro de Informática na Educação (SBIE 2018)

Com base nas repostas coletadas no experimento, foram feitas análises procurando verificar se a hipótese da contribuição do Logos Workbook para o processo de ensino de Lógica Proposicional é aceita na percepção dos usuários. Inicialmente, destaca-se que $100 \%$ dos alunos envolvidos manifestaram que entenderam o propósito do Logos Workbook. Quanto à utilização, $80 \%$ dos alunos concordaram que o sistema é facilmente utilizado ( $40 \%$ concordaram plenamente e $40 \%$ parcialmente), enquanto $20 \%$ discordaram parcialmente dessa afirmação. No que diz respeito à facilitação do processo ensino-aprendizagem vale destacar que para $100 \%$ dos alunos o Logos Workbook contribuiu para este processo (20\% concordaram plenamente e $80 \%$ parcialmente).

Salienta-se ainda, que a comunicação com o professor em caso de dúvida, pelo próprio Logos Workbook também facilitou a aprendizagem: $100 \%$ dos alunos concordam com essa afirmação, $40 \%$ plenamente e $60 \%$ parcialmente. Ainda no que diz respeito ao processo de aprendizagem, 100\% dos alunos concordaram totalmente com a afirmação de que o acesso aos exercícios já resolvidos contribui para a fixação do conteúdo estudado. Por fim, no que tange à identificação de conteúdo postado pelo professor no sistema, $90 \%$ dos alunos concluiram ser facilmente perceptível que a postagem foi realizada pelo docente, enquanto $10 \%$ concordou parcialmente com essa afirmatição. Assim, de modo geral o sistema foi bem avaliado pelos alunos.

\section{Conclusões}

O presente trabalho propôs uma ferramenta de suporte a caderno escolares digitais onde os alunos podem inserir suas anotações, receber conteúdo do professor, e utilizar os recursos já disponíveis para resolver exercícios de Lógica, buscando assim, contribuir para a melhoria no desempenho dos alunos dessa disciplina. Resultados de experimentos empíricos realizados com alunos, trazem evidências que os alunos entenderam o propósito dos cadernos escolares digitais e perceberam sua contribuição no processo de aprendizagem. Como trabalhos futuros, duas possibilidade estão sendo exploradas com o Logos Workbook: a) os dados acumulados da resolução dos exercícios de Lógica durante o decorrer da disciplina, incluindo não apenas o processo de resolução dos alunos, mas as avaliações, diagnósticos e ações pedagógicas do tutor inteligente, podem ser usados para tutoria inteligente no processo de aprendizagem de toda a disciplina e não apenas um exercício por vez, formando o laço mais externo (outer loop - [VanLeh, 2006]) de tutoria do ambiente Heráclito; b) um sistema de suporte ao professores está sendo construído, que irá permitir não apenas o acesso aos cadernos individuais dos alunos, mas uma visão global destes cadernos através de técnicas estatísticas e analíticas.

\section{References}

Albrecht, J. (2017) Using Web-Based Notebooks For Blended-Learning In Computer Science, EDULEARN17 Proceedings, pp. 5714-5720.

Bergmann, J., Sams, A. Flip your classroom: reach every student in every class every day. USA: ISTE, 2012.

Bertram, D. (2016) Likert Scales. 2016, http://poincare.matf.bg.ac.rs/ kristina/topicdane-likert.pdf. 
VII Congresso Brasileiro de Informática na Educação (CBIE 2018)

Anais do XXIX Simpósio Brasileiro de Informática na Educação (SBIE 2018)

Castells, M. (1999) A sociedade em rede. 6a . Edição. São Paulo: Paz e Terra, 1999.

Filipouski, A.M., Marchi, D.M., Simões, L.J. (2009) Lições do Rio Grande - Caderno do Aluno - $1^{\circ}$ Ano do Ensino Médio. Sec. de Estado da Educação, Porto Alegre.

Garrison, D. R., Kanuka, H. (2004) Blended learning: Uncovering its transformative potential in higher education. The Internet and Higher Education, v.7, n.2, p.95-105.

Gluz, J. C., Bueno, R., Peres, R. K., Galafassi, F. P. Tutoria Inteligente Completa para os Conceitos Formais da Lógica Proposicional: Experimentos e Resultado. Anais do SBIE 2017, Recife, PE.

Güzer, B., Caner, H. (2014) The Past, Present and Future of Blended Learning: An in Depth Analysis of Literature. Procedia - Social and Behavioral Sciences, v.116.

Heal, K. M., Hansen, M. L., Rickard, K. M. (1998). Maple V: Learning guide (Série Waterloo Maple). New York: Springer: New York.

Hiratsuka, T.P. (1996) Contribuições da Ergonomia e do Design na Concepção de Interfaces Multimídia. 1996. 153f. Dissertação (Mestrado em Eng. de Produção) Prog. Pós-Grad. em Eng. de Produção, UFSC, Florianópolis, 1996

Marangunić, N., Granić, A. (2015) Technology Acceptance Model: A Literature Review from 1986 to 2013. J. of Univers. Access Inf. Soc. v. 14, n. 1, p. 81-95.

Monagan, M. B. et al. (1998). Maple V: Programming guide (Série Waterloo Maple). New York: Springer.

Nemoto, T. Beglar, D. (2014) Likert-Scale Questionnaires. 2014. https://jaltpublications.org/files/pdf-article/jalt2013_001.pdf .

Rodrigues, A. M.. Galao, M. C., Schwarz, G., Gluz, J. C. (2014) SAAPIENS: Uma ferramenta de Autoria de Objetos de Aprendizagem e Apoio Pedagógico na Dedução Natural na Lógica Proposicional. Anais do SBIE 2014, Dourados, MS.

Santaella, L. (2007) Linguagens líquidas na era da mobilidade. São Paulo: Paulus.

SEE/SP. Secretaria da Educação do Estado de São Paulo. Caderno do aluno, (sociologia). São Paulo: IMESP.

VanLehn, K. (2006). The behavior of tutoring systems. Int. J. of Artificial Intelligence and Education, v.16, p.227-265. 